\section{Uma aplicação do sensoriamento remoto para a investigação de endemias urbanas}

\author{
A remote sensing application to investigate urban \\ endemics
}

\author{
1 Divisão de Processamento \\ de Imagens, Instituto \\ Nacional de Pesquisas \\ Espaciais, São José dos \\ Campos, Brasil. \\ 2 Escola Nacional de Saúde \\ Pública, Fundação Oswaldo \\ Cruz, Rio de Janeiro, Brasil. \\ 3 Instituto de Medicina \\ Social, Universidade do \\ Estado do Rio de Janeiro, \\ Rio de Janeiro, Brasil. \\ ${ }^{4}$ Núcleo de Estudos de \\ Saúde Coletiva, Universidade \\ Federal do Rio de Janeiro, \\ Rio de Janeiro, Brasil. \\ Correspondência \\ V. R. M. Correia \\ Divisão de Processamento de \\ Imagens, Instituto Nacional \\ de Pesquisas Espaciais. \\ Av. dos Astronautas 1758, \\ C. P. 515, São José dos \\ Campos, $S P$ \\ 12201-027, Brasil. \\ virginia@dpi.inpe.br
}

\begin{abstract}
This paper presents a case study on environmental aspects related to the occurrence of visceral leishmaniasis in Teresina, Piauí, Brazil, from 1993 to 1996, in order to discuss the use of some appropriate geo-processing methods for medianresolution remote sensing images potentially useful for studying vector-borne transmissible diseases in urban areas. We present the main techniques: registration, geometric correction, restoration, fusion, segmentation, and classification. Using intra-class correlation indices applied to the proportion of area by class in the census tract, we compare four classifiers: Maxver, Bhattacharya, $K$-means, and Isoseg. This comparison was not devised to choose the best classifier, but to depict different classification scenarios aimed at recognizing the best identifiable image classes in urban settings. We conclude that even with limited resources, using low-cost and easily available median resolution images and free software to process and integrate information, it is possible to identify land use characteristics, potentially appropriate for analyzing urban areas exposed to environmental risk for vector-borne diseases.
\end{abstract}

Remote Sensors; Visceral Leishmaniasis; Endemic Diseases
Virginia Ragoni de Moraes Correia 1,2 Antônio Miguel Vieira Monteiro 1

Marilia Sá Carvalho 2

Guilherme Loureiro Werneck 3,4

\section{Introdução}

A ocorrência de doenças endêmicas transmitidas por vetores caracteriza-se por padrões espaçotemporais distintos. Aspectos do meio físico-biótico, que chamamos aqui de ambientais - regime de chuvas, temperatura, vegetação, coleções hídricas, relevo e geomorfologia, por exemplo - estão associados à proliferação das populações de reservatórios, vetores e agentes infecciosos 1 . Em estudos ecológicos é importante revelar os relacionamentos dos padrões de ocorrência de doenças com estas variáveis de caracterização ambiental. A presença ou ausência de cobertura vegetal vem sendo a característica mais freqüentemente utilizada nos estudos que buscam relacionar ambiente e doenças endêmicas, por seu papel na manutenção do ciclo biológico de vetores e agentes infecciosos, uma vez que esta variável é de resposta relativamente rápida, quando da alteração de outras variáveis ambientais como a precipitação, temperatura e umidade ${ }^{2}$. A relação entre características descritoras da paisagem ambiental e ocorrências de doenças endêmicas é mediada pela estrutura demográfica da população e pelo perfil sócio-econômico local, atuando sobre a suscetibilidade e as possibilidades de contato com vetores e reservatórios 3,4. O uso de sensoriamento remoto por imageadores orbitais se apresenta como uma possibilidade metodológica, que permite a caracterização das variáveis ambientais de interesse em estudos de endemias. 
As técnicas de classificação automática e semi-automática de imagens de sensoriamento remoto permitem ampliar e acelerar o processo de aquisição de informações ambientais relevantes no contexto dos estudos enfocando a interação ambiente-doença. Com o uso dos recursos de Sistemas de Informações Geográficas (SIG), como ambiente computacional para integração dessas variáveis com outras relativas à ocorrência da doença, como os dados de caracterização do perfil sócio-econômico-demográfico, é possível montar um painel sócio-territorial ampliado, que potencializa e amplia o olhar do epidemiologista sobre potenciais focos e áreas de risco.

O interesse recente da saúde pública nas possibilidades de uso do sensoriamento remoto para o estudo de endemias tem se concentrado nas áreas rurais 1 . Entretanto, devido ao processo de urbanização de diversas endemias, como a leishmaniose cutânea e visceral, é oportuno explorar o uso potencial dessas imagens em áreas urbanas e peri-urbanas. A questão urbana é mais complexa e requer o uso de resoluções mais finas e algoritmos de classificação mais especializados. Entretanto, mesmo em médias resoluções, usando recursos de processamento de imagens, é possível detectar cenários de risco pela textura observada como, por exemplo, áreas de ocupação urbana mais ou menos organizada, regiões muito densas ou com maior cobertura de vegetação, aglomerados populacionais próximos a coleções de água ou a terras recém-desmatadas. Essas características formam mosaicos que podem traduzir a dinâmica da interação entre hospedeiros, vetores e agentes infecciosos 1 .

A seguir apresentamos uma breve revisão dos métodos de pré-processamento das imagens de sensoriamento remoto de média resolução, necessários para os procedimentos de classificação aqui utilizados, e para sua integração, em SIG, com outras fontes de informação em áreas urbanas. Os principais procedimentos adotados são apresentados, de forma simplificada, de modo a permitir ao especialista na área de saúde uma visão geral de como utilizar o dado de sensoriamento remoto em seus estudos. Esse item inclui um fluxograma descrevendo os dados de entrada e as operações necessárias para extração da informação relevante no contexto do estudo. Um método para analisar a concordância dos mapas temáticos gerados a partir dos classificadores de imagem por região (Bhattacharya e Isoseg) e por pixel (Maxver e K-médias) é apresentado, e seus resultados analisados e discutidos observando o contexto do estudo de caso em Teresina, Piauí. Esperamos, a partir de uma aplicação relevante, discutir a aplicabilidade de um conjunto de técnicas ligadas ao sensoriamento remoto em média resolução no estudo de endemias transmitidas por vetores em áreas urbanas.

\section{A aplicação do sensoriamento remoto em áreas urbanas}

O resultado dos procedimentos de classificação é um mapa temático, onde cada classe é definida pelo usuário no contexto de sua aplicação; neste estudo visando a indicar situações que potencializem a transmissão de leishmaniose visceral. A indicação parte do conhecimento prévio, de especialistas, que identifica as classes conforme a probabilidade de contato entre população humana, vetores e reservatórios.

Em área urbana, o uso de imagens de sensoriamento remoto de média resolução e de classificadores é um desafio. As dificuldades são proporcionais ao nível de detalhe que se deseja observar, ou seja, as classes temáticas desejadas para o estudo, e às limitações impostas pelas resoluções espaciais e espectrais dos sensores. Existe um grande empenho no desenvolvimento de algoritmos que possam ser mais adequados à complexidade existente 5 . Encontra-se na literatura o uso de metodologias híbridas, em que dois ou mais classificadores são utilizados; classificadores por regra de decisão, em que padrões diferentes podem ser separáveis com base em uma ou mais características 6; os que incorporam características adicionais como textura 7 ; os que utilizam algoritmos $f u z z y^{8}$. No entanto, para os objetivos deste artigo nos concentraremos em métodos de classificação semi-automática de imagens de sensoriamento remoto, consolidados e há muito utilizados em estudos nas áreas ligadas às Ciências Ambientais e às Ciências da Terra em geral.

Classificadores de imagens de sensoriamento remoto podem ser vistos a partir de dois enfoques ${ }^{8}$. O primeiro observa cada pixel isoladamente, realizando uma classificação por pixel, ou, alternativamente, utilizando o conceito de região, conjuntos de pixels definidos por uma propriedade de similaridade. O segundo enfoque refere-se ao fato de os classificadores serem supervisionados, exigindo uma fase de treinamento anterior à classificação, ou não-supervisionados, baseado unicamente nos dados (os pixels que formam a imagem). Os classificadores supervisionados passam por um processo de treinamento em que o usuário faz uso de informações que permitem a identificação de suas classes de interesse. Os não-supervisionados utilizam algoritmos de agrupamentos. Embora automáticos, com pouca participação do investigador, nesta fase, os métodos não-supervisionados precisam 
passar pelo processo conhecido como rotulação. É na rotulação que o especialista então decide que a cada pixel ou região classificada, uma determinada classe temática, do domínio do problema, será atribuída.

$\mathrm{Na}$ área da saúde, a dimensão em estudo casos de doença - é estimada por meio de contagens de casos por área, freqüentemente o setor censitário, considerada uma unidade de análise de tamanho adequado ${ }^{9}$, abrangendo população em torno de 800 pessoas 10 . Na imagem de sensoriamento remoto não vemos setores censitários, o que vemos é a cidade como um contínuo com os elementos que a constitui: casas, ruas, praças, parques etc. Uma etapa importante é sobrepor, em um mesmo ambiente computacional, os dados relativos aos setores e a imagem. Isso é feito em um SIG. Para fazê-lo, alguns procedimentos são necessários, uma vez que em médias resoluções alguns detalhes podem não estar visíveis, o que dificulta a sobreposição. Para fazer um bom ajuste dos setores na imagem, os procedimentos de restauração e fusão, que serão comentados adiante, são utilizados, fazendo com que a imagem de $30 \mathrm{~m}$ possa ser observada com $10 \mathrm{~m}$ e com $5 \mathrm{~m}$, com alguma degradação, mas realçando características importantes como traçado de ruas, permitindo um melhor ajuste dos setores sobre a imagem. Após esse ajuste, utilizando os setores sobrepostos à imagem, observa-se uma heterogeneidade na composição dos setores. Alguns são grandes e pouco urbanizados, outros pequenos e muito densos; outros setores apresentam uma ocupação claramente desorganizada, outros possuem uma ocupação planejada, e outros ainda apresentam composição dessas formas de ocupação, acrescidas de pouca cobertura vegetal e poucos parques; e por fim, vemos setores com uma ocupação organizada e arborização. Esses padrões de ocupação podem estar associados à ocorrência de endemias. Para testar essas hipóteses e quais dessas variáveis de fato participam do processo de estabelecimento e manutenção da endemia, é necessário aferir essas variáveis. Este artigo mostra como essas variáveis podem ser obtidas, extraindo da imagem, por meio do processo de classificação, a variedade de classes temáticas representativas de padrões de uso e cobertura do solo urbano, e distribuindo essas classes em cada um dos setores censitários.

As classificações semi-automáticas com base nas imagens de sensoriamento remoto estão sujeitas a erros. Estes são devido à interpretação feita pelo especialista; às características de resposta espectral semelhante para diferentes elementos da paisagem urbana; à resolução espacial do sensor; à falta de dados coletados em campo para uma verificação mais apurada dos resultados gerados pelos classificadores, entre outros. É desejável que esses erros de classificação sejam quantificados. Para isso é necessário comparar cada classificação com uma de referência, muitas vezes baseada em inspeção local, o que nem sempre é possível. Neste trabalho, não fazemos uma avaliação do resultado da classificação com referência a dados de inspeção local. É feita uma comparação entre os mapas temáticos gerados pelos classificadores, a partir de uma medida de correlação intraclasse, e da avaliação dos cenários gerados com o uso de um conjunto de fotos aéreas da região (analógicas).

\section{Etapas envolvidas no processamento das imagens}

O registro de imagem refere-se ao ajuste dos sistemas de coordenadas de duas imagens de uma mesma área, utilizando-se um dos sistemas como referência. Para integrar imagens de diferentes períodos ou diferentes sensores essa operação é necessária. Define-se correção geométrica como a transformação de uma imagem de sensoriamento remoto à escala e projeção de um mapa de referência 11. Para que as imagens possam ser integradas aos SIG essas operações são necessárias 6 .

Os registros podem ser manuais ou automáticos. Para o registro manual é necessário identificar pontos de controle no terreno usando sistemas de posicionamento global (global positioning system-GPS) 6 . O registro automático utiliza algoritmos para detecção de pontos de controle baseados na correlação entre as duas imagens com mínima interferência do usuário 12.

As técnicas de restauração visam a modelar o processo de degradação radiométrica a fim de obter imagens com menos ruído e maior nível de detalhes. Para essa operação são utilizados filtros lineares cujos pesos são obtidos a partir das características do sensor e banda espectral 13 .

A operação de fusão tem por objetivo principal integrar informação de diferentes sensores, a fim de se obter uma imagem com melhor resolução espacial e espectral. A fusão é muito utilizada quando o sensor é rico em características espectrais, e possui uma banda pancromática com resolução melhor, como os sensores do Landsat ETM-7, o SPOT e o Ikonos. Dentre algumas técnicas de fusão, a mais comum é a que utiliza uma transformação no espaço de cores conhecida por IHS 6,14.

A segmentação é uma operação que divide a imagem em regiões compostas por conjuntos de pixels que apresentam propriedades semelhan- 
tes como: valor de sua resposta espectral próximo, textura etc. 15 .

A classificação supervisionada é aquela que necessita de um treinamento no qual o usuário determina, por meio de amostras, características do objeto que se quer mapear. Esses objetos ou classes devem ser definidos a priori e sua identificação é limitada pelas características espaciais, espectrais do sensor utilizado. Pode-se usar como área de treinamento uma região delimitada pelo usuário ou algumas regiões da imagem segmentada, já que estas anteriormente determinaram regiões homogêneas, segundo o critério de semelhança utilizado. Dessas amostras de treinamento calculam-se parâmetros para cada classe.

O classificador supervisionado por máxima verossimilhança, Maxver, é o mais utilizado em sensoriamento remoto ${ }^{6}$. É uma classificação pontual, baseada no valor radiométrico do pixel e nos parâmetros da distribuição Gaussiana de cada uma das classes de treinamento. O mapa obtido por esse classificador, em geral, tem uma aparência ruidosa, visto que o contexto espacial não é considerado.

A classificação Bhattacharya, por outro lado, utiliza amostras de alguns segmentos para treinar o classificador, calculando a média e matriz de covariância de cada classe. Em seguida, cada segmento é alocado a uma dessas classes segundo a menor distância de Bhattacharya 16 .

Na classificação não-supervisionada as classes não são definidas a priori e, portanto, uma operação de rotulação pós-classificação se faz necessária. O classificador não-supervisionado denominado Isoseg 15, baseia-se no agrupamento de regiões similares caracterizadas pela média e pela matriz de covariância. O número de classes é dependente do limiar de aceitação determinado pelo usuário. Esse limiar define uma distância máxima de Mahalanobis. Regiões cujas médias estiverem dentro do hiperelipsóide, definido pelo limiar de aceitação, pertencem a uma certa classe. O $K$-médias é um classificador não - supervisionado que utiliza uma abordagem de agrupamento. O espaço de atributos da imagem é partido em K grupos. A partir dessa situação inicial, cada pixel da imagem é alocado ao centro mais próximo segundo a distância euclidiana 6 .

Uma matriz de erro é uma forma de representar a precisão de um mapa temático. As colunas da matriz representam o dado de referência e as linhas o resultado da classificação. Os valores na diagonal dessa matriz, geralmente contendo número de pixels, representam a concordância entre o classificador e a referência. A precisão individual de cada categoria pode ser analisada via erros de inclusão, ou seja, incluir uma área numa categoria que ela não pertence, e através de erros exclusão, qual seja, excluir uma área de uma categoria a qual ela pertença. Além desses erros é possível calcular também medidas como acurácia total, acurácia do produtor e acurácia do usuário 17 .

Uma medida de concordância comumente utilizada é a estatística kappa 18. Essa medida, utilizada para dados categóricos, é definida como a proporção de concordância observada não decorrente do acaso, em relação à máxima concordância não devida ao acaso ${ }^{19}$. Valores possíveis para kappa variam de $-1 \mathrm{a}+1$. Espera-se uma correlação positiva entre o dado de referência e o da classificação e um valor maior que a concordância ao acaso, representada pelo valor 0 .

Para dados contínuos, uma medida de concordância é o ICC (coeficiente de correlação intraclasse). O ICC estima a proporção da variabilidade total devida à variação entre os classificadores e também varia de $-1 \mathrm{a}+1$. Landis \& Koch 20 propõem a seguinte classificação para interpretação dos resultados de análises baseadas nas estatísticas kappa e/ou ICC: quase perfeita (> 80\%), substancial (entre $60 \%$ e $80 \%$ ), moderada (entre $40 \%$ e $60 \%$ ), fraca (entre $20 \%$ e $40 \%$ ), quase nenhuma $(<20 \%) 21$.

\section{Material e método}

\section{Área de estudo}

Teresina, capital do Estado do Piauí, localiza-se na confluência dos rios Parnaíba e Poti, centralizada a $5^{\circ} 5^{\prime}$ de Latitude Sul e na Longitude Oeste de $42^{\circ} 48^{\prime}$. A região de estudo abrange a área urbana da cidade e a correspondente malha de setores censitários do ano de 1991. A cidade tem um clima tropical com temperatura média anual de $27^{\circ} \mathrm{C}$ e precipitação pluviométrica média anual de $1.300 \mathrm{~mm}$. A vegetação predominante consiste de árvores esparsas e arbustos, existindo nas periferias da cidade regiões de pastagem e floresta tropical. O uso do solo na região é predominantemente urbano residencial, comercial e industrial. Na cidade de Teresina, uma das capitais mais pobres do Brasil, a leishmaniose visceral é um problema de saúde pública. Em 1980 houve a primeira epidemia urbana, com número de casos crescente até 1985, declinando nos anos seguintes até início de uma nova epidemia na década de 90, quando registrou-se um número de casos, novos, maior que 1.200. 
Para o estudo utilizou-se uma imagem LandsatTM5, passagem em 17 de agosto de 1990, e uma Landsat-ETM7 de 6 de julho de 2001, órbita 219, ponto 64, projeção UTM. A imagem de 1990 foi escolhida por ter uma data próxima a do censo demográfico. A de 2001, por conter uma banda pancromática com $15 \mathrm{~m}$ de resolução, foi utilizada para possibilitar o registro dos setores censitários à imagem em ambiente SIG.

Foram utilizadas as bandas 3 (vermelho), 4 (infravermelho próximo) e 5 (infravermelho médio), em acordo com a literatura para estudos urbanos, por serem as bandas, no Landsat, que permitem acomodar melhor as respostas espectrais em alvos urbanos, nesta resolução ${ }^{22}$. O período escolhido foi o de seca, pela facilidade de se obter imagens sem cobertura de nuvens.

Utilizou-se o programa SPRING versão 4.1 (Sistema de Processamento de Informações Georeferenciadas; http://www.dpi.inpe.br/ spring) 23.

\section{Processamento e análise}

A Figura 1 ilustra as principais etapas da metodologia utilizada. A imagem foi segmentada para aplicações dos classificadores Bhattacharya e Isoseg. Para o treinamento da classificação supervisionada e a rotulação da classificação não-supervisionada, utilizou-se um conjunto de amostras com fotos aéreas e informações de especialista que desenvolve trabalho de campo na região.

A imagem de 1990 foi utilizada para a classificação visando a posterior análise de casos da doença entre 1993 e 1996. A de 2001, única ETM-7, com a banda pancromática de $15 \mathrm{~m}$, passou pela operação de restauração e fusão com a finalidade de se obter uma imagem colorida com $5 \mathrm{~m}$ de resolução, para o registro do setor censitário à imagem. Sendo a imagem de 2001 e os setores de 1991, cruzamentos de ruas foram utilizados como referência para os ajustes. A sobreposição dos setores censitários de 1991 à imagem, permitiu o cálculo da proporção de cada classe temática definida em cada setor.

Diversas segmentações, com variação dos parâmetros de similaridade e de área, foram apresentadas ao especialista para seleção dos padrões de interesse, que escolheu a combinação similaridade $=12$ e área $=50$ para as classificações Isoseg e Bhattacharya. Na Figura 2, para ilustrar os diferentes resultados que podem ser obtidos nessa operação, é apresentado o resultado das segmentações com duas combinações de similaridade e área.
No treinamento dos classificadores supervisionados e rotulação dos não-supervisionados foram utilizadas as amostras ilustradas na Figura 3 , e descritas na Tabela 1 . As classes foram escolhidas de forma a priorizar certos padrões espaciais importantes para a ocorrência da endemia, definidas pelo especialista, independente de conhecimento prévio dos algoritmos.

Para o treinamento do classificador Bhattacharya as amostras foram superpostas aos segmentos. Na etapa de rotulação, referente aos classificadores K-médias e Isoseg, utilizou-se o conjunto de amostras. Para o método Maxver, algumas amostras foram utilizadas para adquirir os parâmetros estatísticos para cada classe, e outras separadas para serem usadas como amostras-teste, possibilitando uma avaliação prévia da classificação por meio do índice kappa.

Um programa utilizando a linguagem LEGAL 24 foi desenvolvido para calcular a proporção de cada classe nos setores censitários, para cada um dos classificadores. Os classificadores foram comparados pelo coeficiente de correlação intraclasse.

\section{Resultados}

O classificador Maxver foi avaliado utilizando-se as amostras teste, obtendo-se acurácia total de $71,68 \%$ (Tabela 2). Para as classes água, solo exposto e vegetação densa, a acurácia em relação ao padrão de referência (amostras de treinamento) é alta e para as diversas classes residenciais a acurácia foi apenas moderada. Os resultados obtidos para as classes residenciais com muita vegetação, com vegetação moderada e esparsa muito verde foram ligeiramente superiores a $40 \%$. Houve confusão na classificação das amostras das duas primeiras classes mencionadas, e entre a residencial esparsa muito verde com a vegetação densa e a agricultura. Esse desempenho, no caso do classificador Maxver, pode ser explicado pelas dificuldades em estabelecer essas classes temáticas nessa resolução espacial, considerando a limitação para discriminar alvos com uma mistura de vários elementos em diferentes proporções, que causam impacto na resposta espectral e, portanto, na caracterização desses temas. Além disso, o classificador Maxver supõe uma distribuição Gaussiana para as classes e, algumas amostras obtidas apontavam para uma distribuição bimodal. O valor de kappa foi de $67,62 \%$, ou seja, uma concordância moderada. O resultado visual do classificador Maxver é apresentado na Figura 4a.

O classificador Bhattacharya se aplica sobre o resultado do processo de segmentação 16 , ou 


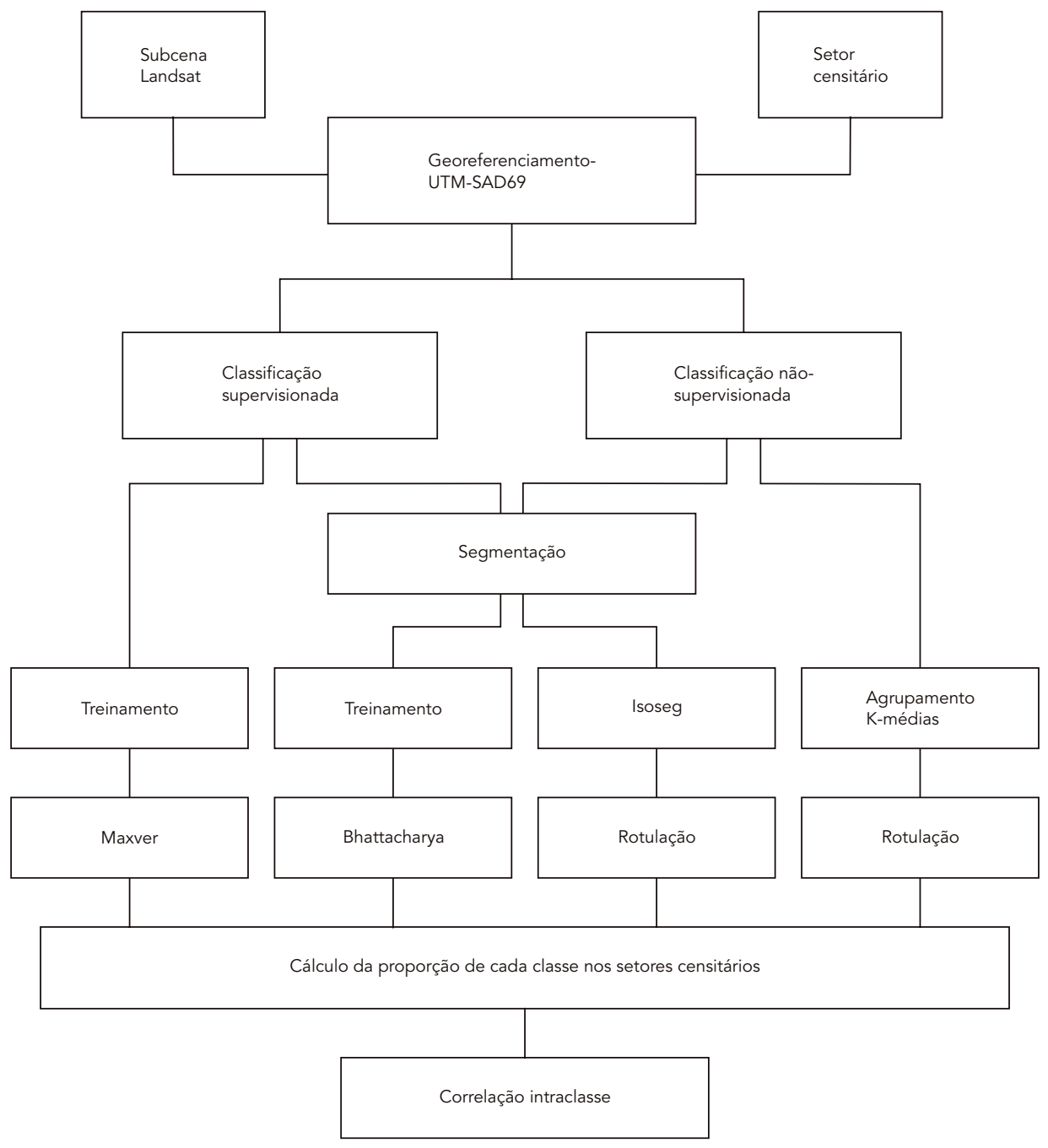

seja, classifica-se um conjunto de pixels, gerando áreas mais contínuas, de no mínimo 50 pixels (Figura 4b).

O classificador Isoseg apresentou resultado semelhante ao Bhattacharya, gerando 19 classes. Para o processo de rotulação buscou-se interseções entre as classes encontradas e as amostras. Esse processo é lento e demanda uma boa experiência e familiaridade com a interpretação de imagens de sensoriamento remoto. As áreas brancas no mapa (Figura 4c) mostram as áreas onde a rotulação não foi possível.

Para o algoritmo K-médias, também não-supervisionado, só foi possível rotular seis classes
(Figura 4d). Foram geradas 11 classes a partir das definições do especialista, embora, dado o tamanho da área, o número esperado de classes seja menor ${ }^{25}$. No processo de rotulação as dificuldades foram semelhantes às do Isoseg.

A Tabela 3 mostra o ICC entre classificadores por classe e o ICC médio para cada combinação de classificadores. O K-médias não foi considerado nessa avaliação dada a diferença no número de classes. No cálculo do ICC médio não foram incluídas as classes "residencial com vegetação moderada" e "residencial esparsa muito verde" por não terem sido detectadas pelo classificador Isoseg. O ICC médio foi moderado para pratica- 
2a) Segmentador com parâmetros de similaridade $=8 \mathrm{e}$ área $=20$

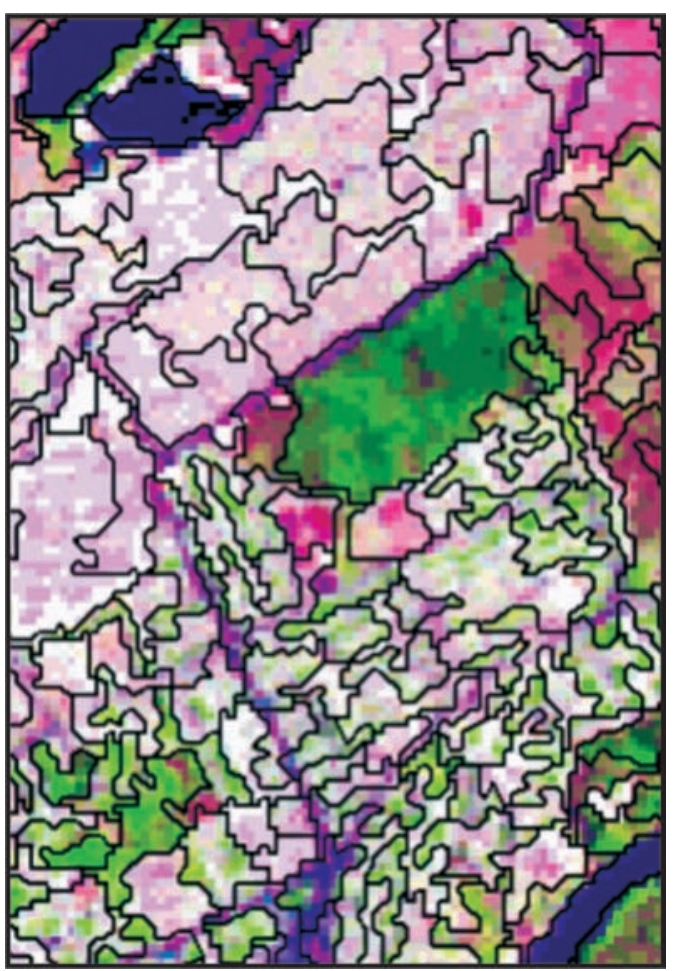

2b) Segmentador com parâmetros de similaridade $=12 \mathrm{e}$ área $=50$.

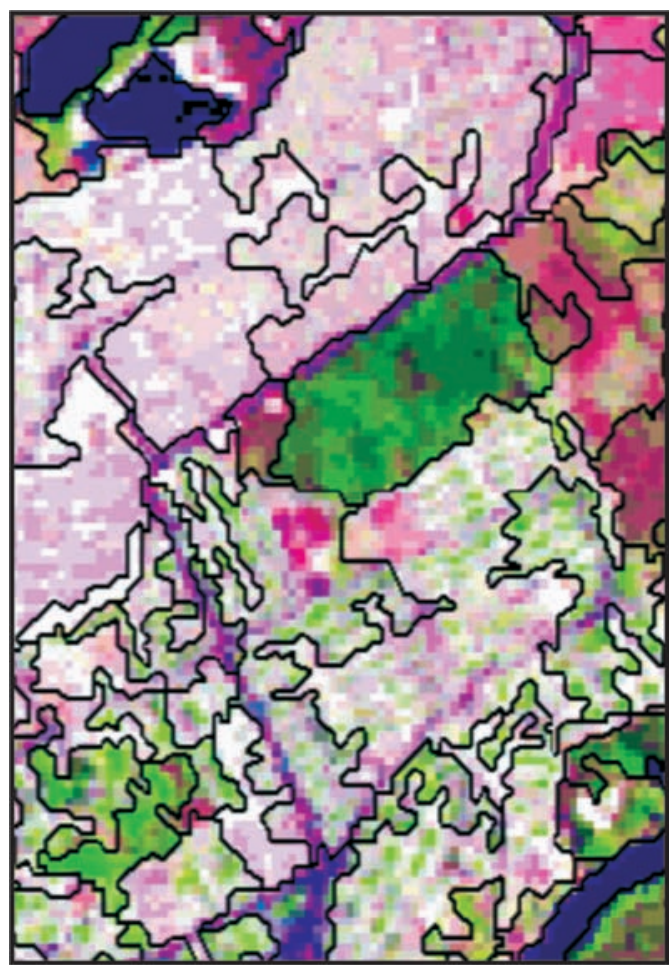

mente todas as combinações de classificadores. As classes "água" e "vegetação densa" apresentaram um valor de ICC quase perfeito para algumas combinações, confirmando que são bem detectadas por todos os classificadores. As classes "área descoberta", "industrial ou comercial" e "residencial de alta densidade" tiveram um ICC acima de $60 \%$. As áreas descobertas eram poucas, mas foram detectadas similarmente pelos classificadores. As classes "industrial ou comercial" e "residencial de alta densidade" ocuparam uma área razoável, a primeira mais concentrada na parte central do mapa e a segunda espalhada, mas com três agrupamentos com uma concordância muito boa. "Solo exposto" apresentou ICC muito baixo em geral, e somente em dois grandes setores ao norte foram detectadas. A classe "banco de areia" quase não ocorre na imagem e o valor do ICC foi substancial apenas para a combinação Maxver e Bhattacharya, classificadores supervisionados. O classificador Isoseg detectou presença de "banco de areia" em setores para os quais essa classe estava ausente nos outros classificadores. O ICC das áreas "residencial com vegetação moderada", "residencial com muita vegetação" e "residencial esparsa muito verde" reflete a dificuldade dos classificadores em separar essas classes. Entre os classificadores Isoseg e Bhattacharya o ICC foi substancial na classe "residencial com muita vegetação". Os valores baixos para as outras combinações podem ser atribuídos ao baixo desempenho do Maxver na detecção dessa classe. As classes "residencial esparsa muito verde" e a "residencial com vegetação moderada" apresentaram um ICC moderado para a combinação Maxver e Bhattacharya. Como o Isoseg não detectou essas duas classes, o ICC é nulo. Quanto à área de agricultura, o 
Figura 3

Classes de amostras utilizadas para treinamento dos classificadores supervisionados e rotulação dos não-supervisionados.

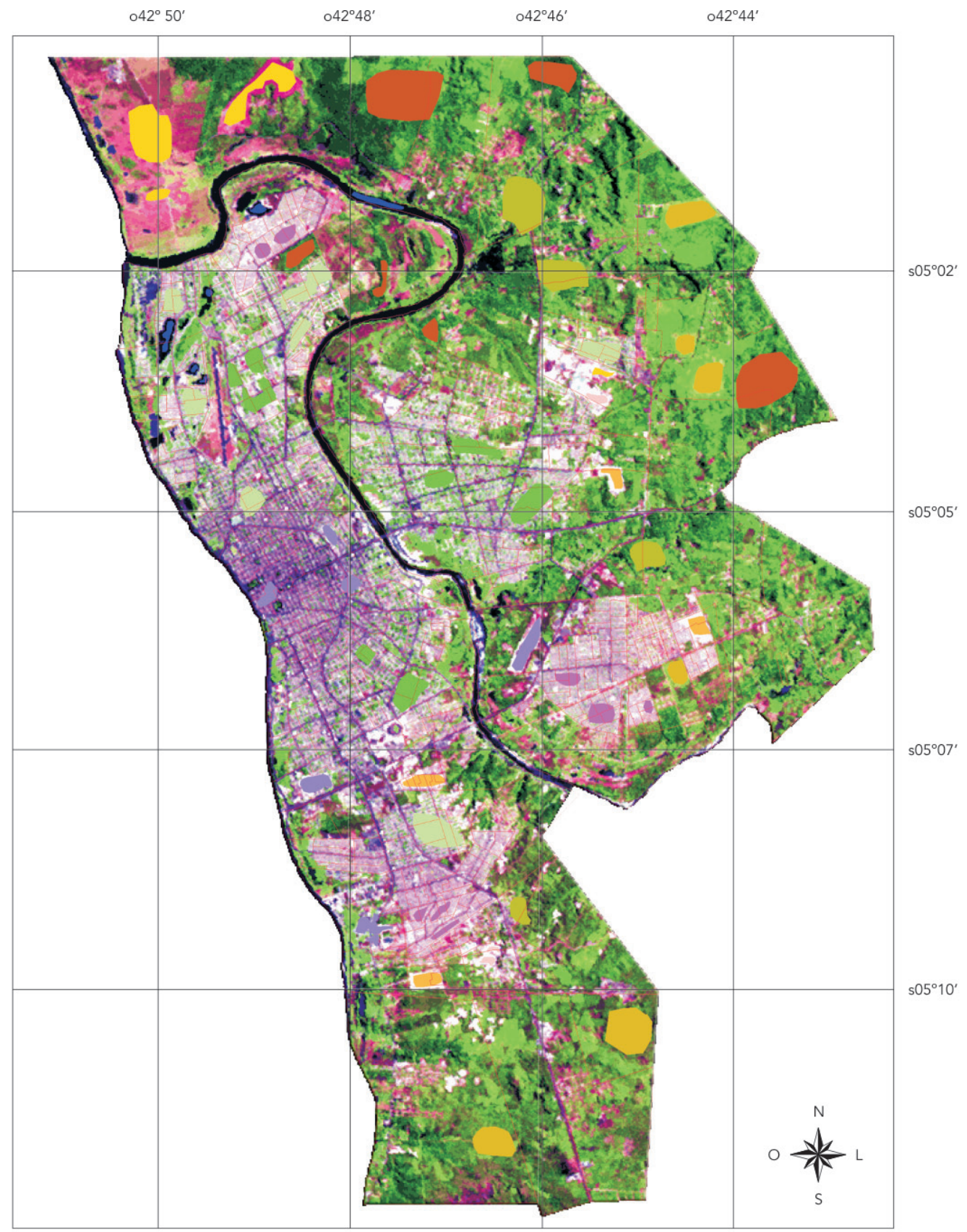

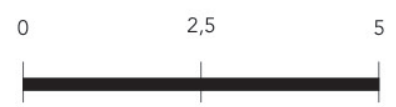

Quilômetros

Area construída descoberta
Banco de areia
Agricultura
Solo

Vegetação densa

Residencial esparsa muito verde

Residencial muito verde

Industrial e comercia
Residencial verde

Residencial densa

Água 
Classes, definições, número de amostras para cada classe, número de pixels para aquisição e teste.

\begin{tabular}{|c|c|c|c|c|c|}
\hline \multirow[t]{2}{*}{ Classes [Denominação utilizada (código)] } & \multirow[t]{2}{*}{ Amostras } & \multicolumn{3}{|c|}{ Pixels/Amostras } & \multirow[t]{2}{*}{ Definição das classes temáticas } \\
\hline & & Total & Aquisição & Teste & \\
\hline \multicolumn{6}{|l|}{ Residencial de alta densidade } \\
\hline [Residencial densa (RD)] & 8 & 987 & 672 & 315 & Área residencial sem cobertura vegetal \\
\hline \multicolumn{6}{|l|}{ Residencial com vegetação moderada } \\
\hline [Residencial verde (RV)] & 7 & 2.674 & 2.159 & 515 & Área residencial com cobertura vegetal baixa \\
\hline \multicolumn{6}{|l|}{ Residencial com muita vegetação } \\
\hline [Residencial muito verde (RMV)] & 8 & 2.481 & 1.779 & 702 & Área residencial com cobertura vegetal alta \\
\hline \multicolumn{6}{|l|}{ Residencial esparsa muito verde } \\
\hline [Residencial esparsa muito verde (REMV)] & 4 & 2.616 & 1.843 & 773 & $\begin{array}{l}\text { Residências em áreas rurais onde a cobertura vegetal } \\
\text { é densa }\end{array}$ \\
\hline Vegetação densa [Vegetação densa (VD)] & 6 & 4.709 & 3.967 & 742 & Grandes áreas com cobertura vegetal densa \\
\hline Industrial ou comercial [Indústria comércio (IC)] & 6 & 1.258 & 854 & 404 & Região central com alta densidade de ruas \\
\hline Solo exposto [Solo (S)] & 4 & 2.114 & 936 & 1.178 & Solo exposto \\
\hline Agricultura [Agricultura (AG)] & 6 & 3.068 & 2.420 & 648 & Áreas agrícolas \\
\hline Banco de areia [Banco de areia (BA)] & 3 & 150 & 125 & 25 & Áreas arenosas presentes nos rios \\
\hline Área descoberta [Área construída descoberta (AD)] & 4 & 736 & 595 & 141 & Áreas desmatadas para construções ou plantações \\
\hline Água $[$ Água $(A)]$ & 6 & 355 & 244 & 111 & Coleções hídricas, lagos, rios etc. \\
\hline
\end{tabular}

Tabela 2

Avaliação da classificação Maxver utilizando amostras teste.

\begin{tabular}{|c|c|c|c|c|c|c|c|c|c|c|c|c|}
\hline Classes & $\mathrm{RD}$ & RV & RMV & REMV & VD & IC & $S$ & AG & BA & $A D$ & A & Total \\
\hline \multirow[t]{2}{*}{ RD } & 282 & 20 & 0 & 0 & 0 & 1 & 1 & 0 & 0 & 11 & 0 & 315 \\
\hline & $89,52 \%$ & $6,35 \%$ & $0,00 \%$ & $0,00 \%$ & $0,00 \%$ & $0,32 \%$ & $0,32 \%$ & $0,00 \%$ & $0,00 \%$ & $3,49 \%$ & $0,00 \%$ & $100,00 \%$ \\
\hline \multirow[t]{2}{*}{ RV } & 81 & 210 & 92 & 36 & 0 & 85 & 4 & 2 & 0 & 5 & 0 & 515 \\
\hline & $15,73 \%$ & $40,78 \%$ & $17,86 \%$ & $6,99 \%$ & $0,00 \%$ & $16,51 \%$ & $0,78 \%$ & $0,39 \%$ & $0,00 \%$ & $0,97 \%$ & $0,00 \%$ & $99.99 \%$ \\
\hline \multirow[t]{2}{*}{ RMV } & 83 & 170 & 294 & 127 & 1 & 15 & 3 & 6 & 0 & 3 & 0 & 702 \\
\hline & $11,82 \%$ & $24,22 \%$ & $41,88 \%$ & $18,09 \%$ & $0,14 \%$ & $2,14 \%$ & $0,43 \%$ & $0,85 \%$ & $0 \%$ & $0,43 \%$ & $0,00 \%$ & $100,00 \%$ \\
\hline \multirow[t]{2}{*}{ REMV } & 6 & 30 & 28 & 351 & 181 & 1 & 8 & 168 & 0 & 0 & 0 & 773 \\
\hline & $0,78 \%$ & $3,88 \%$ & $3,62 \%$ & $45,41 \%$ & $23,42 \%$ & $0,13 \%$ & $1,03 \%$ & $21,73 \%$ & $0,00 \%$ & $0,00 \%$ & $0,00 \%$ & $100,00 \%$ \\
\hline \multirow[t]{2}{*}{ VD } & 0 & 0 & 0 & 20 & 721 & 0 & 0 & 1 & 0 & 0 & 0 & 742 \\
\hline & $0,00 \%$ & $0,00 \%$ & $0,00 \%$ & $2,70 \%$ & $97,17 \%$ & $0,00 \%$ & $0,00 \%$ & $0,13 \%$ & $0,00 \%$ & $0,00 \%$ & $0,00 \%$ & $100,00 \%$ \\
\hline \multirow[t]{2}{*}{ IC } & 12 & 67 & 11 & 7 & 0 & 239 & 24 & 1 & 9 & 34 & 0 & 404 \\
\hline & $2,97 \%$ & $16,58 \%$ & $2,72 \%$ & $1,73 \%$ & $0,00 \%$ & $59,16 \%$ & $5,94 \%$ & $0,25 \%$ & $2,23 \%$ & $8,42 \%$ & $0,00 \%$ & $100,00 \%$ \\
\hline \multirow[t]{2}{*}{ S } & 12 & 6 & 0 & 70 & 0 & 25 & 1.028 & 37 & 0 & 0 & 0 & 1.178 \\
\hline & $1,02 \%$ & $0,51 \%$ & $0,00 \%$ & $5,94 \%$ & $0,00 \%$ & $2,12 \%$ & $87,27 \%$ & $3,14 \%$ & $0,00 \%$ & $0,00 \%$ & $0,00 \%$ & $100,00 \%$ \\
\hline \multirow[t]{2}{*}{ AG } & 0 & 2 & 2 & 34 & 0 & 0 & 13 & 597 & 0 & 0 & 0 & 648 \\
\hline & $0,00 \%$ & $0,31 \%$ & $0,31 \%$ & $5,25 \%$ & $0,00 \%$ & $0,00 \%$ & $2,00 \%$ & $92,13 \%$ & $0,00 \%$ & $0,00 \%$ & $0,00 \%$ & $100,00 \%$ \\
\hline \multirow[t]{2}{*}{ BA } & 0 & 0 & 0 & 0 & 0 & 0 & 0 & 0 & 23 & 2 & 0 & 25 \\
\hline & $0,00 \%$ & $0,00 \%$ & $0,00 \%$ & $0,00 \%$ & $0,00 \%$ & $0,00 \%$ & $0,00 \%$ & $0,00 \%$ & $92 \%$ & $0,08 \%$ & $0,00 \%$ & $100,00 \%$ \\
\hline \multirow[t]{2}{*}{$A D$} & 1 & 0 & 0 & 0 & 0 & 9 & 0 & 0 & 6 & 125 & 0 & 141 \\
\hline & $0,71 \%$ & $0,00 \%$ & $0,00 \%$ & $0,00 \%$ & $0,00 \%$ & $6,38 \%$ & $0,00 \%$ & $0,00 \%$ & $4,26 \%$ & $88,65 \%$ & $0,00 \%$ & $100,00 \%$ \\
\hline \multirow[t]{2}{*}{ A } & 0 & 0 & 0 & 0 & 0 & 0 & 0 & 0 & 0 & 0 & 111 & 111 \\
\hline & $0,00 \%$ & $0,00 \%$ & $0,00 \%$ & $0,00 \%$ & $0,00 \%$ & $0,00 \%$ & $0,00 \%$ & $0,00 \%$ & $0,00 \%$ & $0,00 \%$ & $100,00 \%$ & $100,00 \%$ \\
\hline Total & 477 & 505 & 427 & 645 & 903 & 375 & 1.081 & 812 & 38 & 180 & 111 & 5.554 \\
\hline
\end{tabular}

Desempenho geral: 71,68\%; Confusão média: 28,32 \%; kappa: 67,62\%. 
Figura 4

Classificadores.
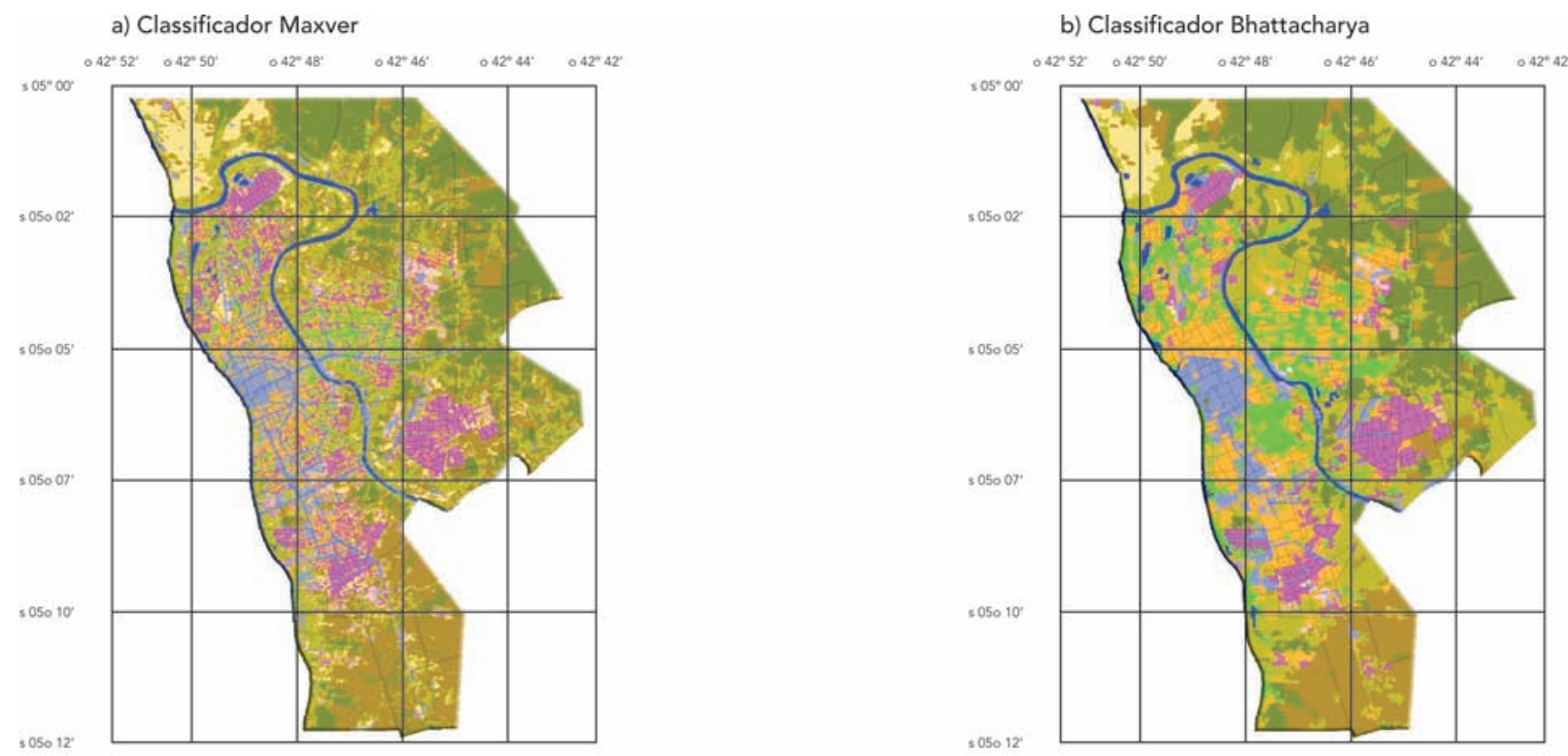

c) Classificador Isoseg

d) Classificador K-médias
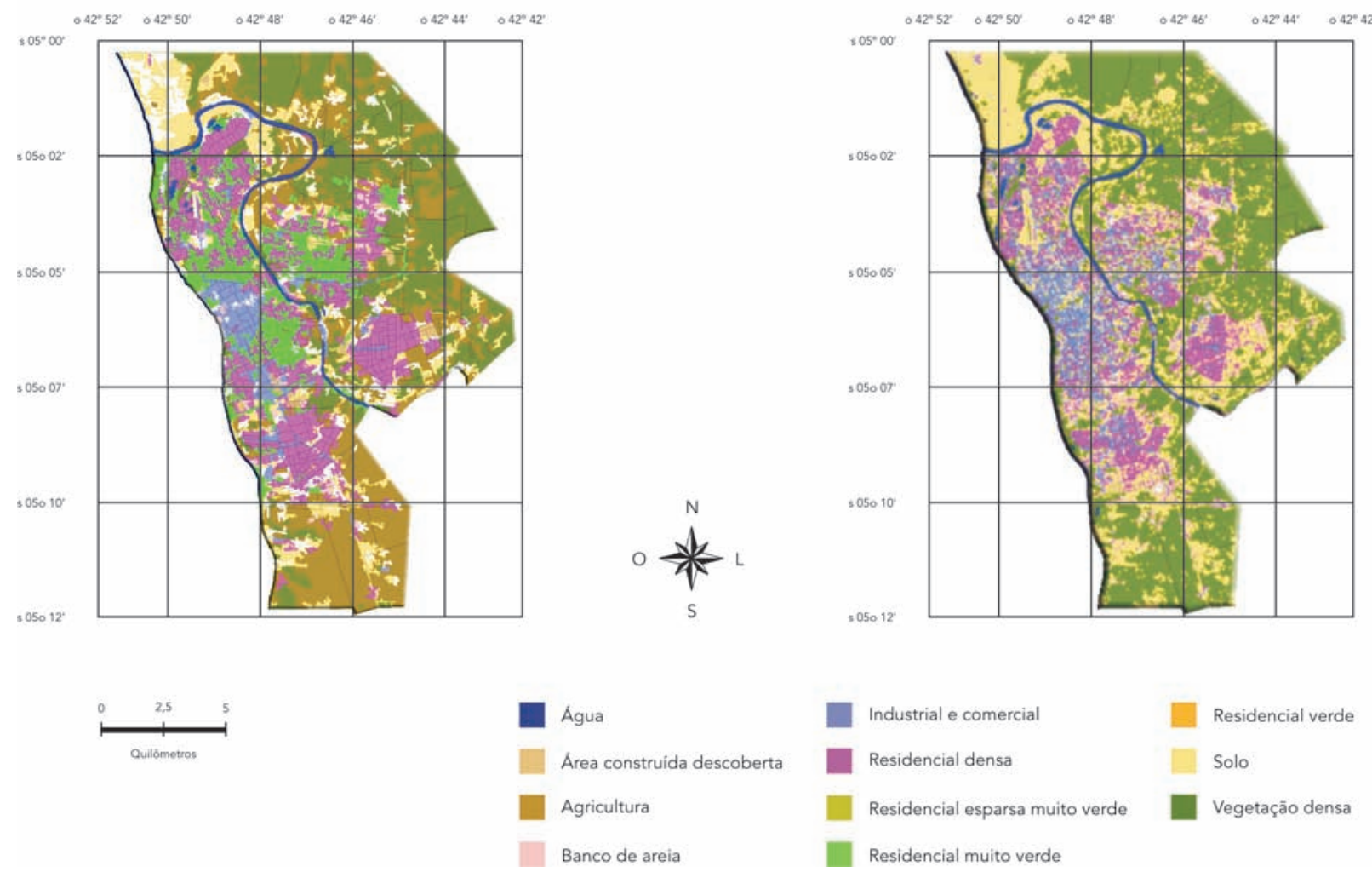

Industrial e comercial

Residencial densa

Residencial verde

Residencial esparsa muito verde

Solo

Vegetação densa

Residencial muito verde 
Coeficiente de correlação intraclasse (ICC) para cada classe e ICC médio para diferentes combinações de classificadores.

\begin{tabular}{|c|c|c|c|c|}
\hline \multirow[t]{2}{*}{ Classes } & \multicolumn{4}{|c|}{ ICC (comparações entre algoritmos) } \\
\hline & $1,2,3$ & 1,2 & 1,3 & 2,3 \\
\hline Água & 0,8275 & 0,7564 & 0,9221 & 0,8346 \\
\hline Vegetação densa & 0,8235 & 0,8910 & 0,7537 & 0,8350 \\
\hline Industrial ou comercial & 0,7320 & 0,6344 & 0,6890 & 0,8490 \\
\hline Residencial de alta densidade & 0,7116 & 0,7849 & 0,7643 & 0,6107 \\
\hline Solo exposto & 0,1040 & 0,1175 & 0,1469 & 0,0168 \\
\hline Banco de areia & 0,1372 & 0,6627 & 0,0855 & 0,1123 \\
\hline Residencial com vegetação moderada & 0,2623 & 0,5247 & 0,0000 & 0,0000 \\
\hline Residencial com muita vegetação & 0,5238 & 0,4286 & 0,3755 & 0,6639 \\
\hline Residencial esparsa muito verde & 0,2998 & 0,5997 & 0,0000 & 0,0000 \\
\hline Agricultura & 0,6254 & 0,5713 & 0,8223 & 0,4062 \\
\hline Área descoberta & 0,8066 & 0,8883 & 0,7458 & 0,7851 \\
\hline Média global & 0,5322 & 0,6236 & 0,5823 & 0,4649 \\
\hline Média global sem a 7ạ e a 9ạ classes & 0,5880 & 0,6372 & 0,5894 & 0,5682 \\
\hline
\end{tabular}

Nota: 1 é o classificador Maxver, 2 é o classificador Bhattacharya e 3 o Isoseg.

resultado da classificação para o Isoseg e o Maxver foi muito semelhante nos setores censitários ao sul, refletindo um valor de ICC quase perfeito. O resultado do Bhattacharya não foi tão bom: classificou como área "residencial esparsa muito verde" uma área classificada como "agricultura" pelos outros classificadores.

Dentre os classificadores avaliados, os mais apropriados seriam os supervisionados: Bhattacharya, com uma segmentação utilizando os limiares 12-50 e o Maxver, que demonstrou um bom desempenho para este caso, mesmo sendo um classificar pontual. O K-médias apresentou o pior desempenho neste contexto e o Isoseg teria tido um bom desempenho sem as restrições da rotulação.

\section{Discussão}

Comparar métodos de processamento de imagem visando ao estudo de endemias urbanas é de grande interesse, pois embora teoricamente discutidos 1, poucos são os trabalhos empíricos sobre o assunto. A utilização do ICC para verificar a concordância por tema, usando o setor censitário como unidade de análise, é uma contribuição importante deste trabalho para avaliação de métodos de classificação. Neste estudo foi utilizada, também, uma nova técnica de registro automático de imagens, mais rápida e tão ou mais eficiente que a manual, com a vantagem de minimizar o viés introduzido pelo operador. Para sobrepor a malha do setor censitário à imagem, dispuse- mos, além do registro vetorial, de ferramentas de restauração e fusão de imagens, sendo possível localizar ruas e cruzamentos, facilitando a aquisição de pontos de controle para o registro 12 .

O resultado global do desempenho dos classificadores mostra grande potencial, mesmo sem a etapa de trabalho de campo. Apesar disso, confirmou-se também as restrições quanto ao uso de classificadores baseados nas informações espectrais de sensores de média resolução em áreas urbanas, mostrando a dificuldade em discriminar padrões importantes para a leihsmaniose, tais como os diversos tipos de solo: urbano, não urbano, com pouca vegetação, áreas agrícolas de áreas rurais com muita vegetação e habitações esparsas 26 .

Os métodos de avaliação utilizados não tiveram o propósito de escolher o melhor classificador. Na realidade eles representam meios para observar cenários de classificação produzidos sem muita informação de coleta e inspeção de campo. As análises permitem emitir um parecer sobre as classes que são mais confiáveis, com valor alto de ICC, assim como as não confiáveis, indicando a necessidade de trabalho e verificação em campo e de melhor amostragem, por exemplo. Essa análise pode sugerir pesos diferentes para as diversas classes temáticas com base na confiança estabelecida a partir das análises do ICC.

Uma forma de melhorar o desempenho dos classificadores é aumentar o número de amostras de qualidade das classes em que a resolução espacial limita a capacidade de discriminar 
adequadamente. Entretanto, aplicações de sensoriamento remoto em saúde, em países em desenvolvimento, dependem da disponibilidade de tecnologias abertas, amplamente disponíveis, de baixo impacto para inserção nos serviços e, com mínimo trabalho de campo para aquisição das variáveis. A precisão, no sentido da exatidão cartográfica do mapeamento, não é o aspecto mais importante, mas sim a preservação das relações entre as localizações obtidas com os outros dados espacializados que fazem parte da análise. Além disso, deve-se ressaltar o acompanhamento do especialista na definição das classes temáticas de interesse, quer seja no caso dos métodos supervisionados, durante o treinamento, quer seja nos métodos não-supervisionados, na fase de rotulação.

É importante frisar que neste estudo nos concentramos no uso potencial de dados de média resolução espacial, imagens entre 20 e 30 metros de resolução, que têm ampla disponibilidade a baixos custos, e têm sido pouco exploradas em estudos de endemias na escala urbana. Não faz parte do escopo deste trabalho a verificação do uso de satélites imageadores mais recentes, com alta e altíssima resolução. Além disso, diversas outras técnicas poderiam ser testadas, tais como novos classificadores, classificações contínuas, análise de mistura, ou mesmo a combinação de outras bandas espectrais além das bandas 3, 4 e 5. Entretanto, a abordagem dessas técnicas não seria possível em apenas um artigo, e fugiria ao objetivo introdutório sobre o tema, ainda pouco explorado na saúde pública.

Neste estudo verificamos que a consideração de um parâmetro global para a partição de toda a área pode trazer prejuízos ao processo classificatório. Uma sugestão seria fazer modificações nos algoritmos de segmentação para que possam re- agir adaptativamente, com base nas estatísticas locais da imagem, e assim acomodar os valores encontrados em cada pixel aos pressupostos estatísticos dos métodos utilizados, alterando dinamicamente os valores dos limiares. Por exemplo, na área urbana, entre os rios, onde a variância é maior, pode-se pensar em aplicar limiares diferentes de áreas e de similaridade.

Transformações ambientais associadas a movimentos migratórios e ao processo de urbanização podem explicar, em parte, porque a leishmaniose visceral, originalmente uma doença restrita às áreas rurais, passou a ocorrer de forma endêmica e epidêmica em grandes cidades brasileiras 27,28. Este processo desordenado de ocupação urbana resultou em condições precárias de vida e destruição ambiental, fatores que também podem ter influenciado a emergência da doença no meio urbano. Acreditamos que o uso do sensoriamento remoto possa contribuir para elucidar o papel desempenhado por esse processo socioambiental complexo que vem sendo associado à emergência da leishmaniose visceral no meio urbano, e conseqüentemente, aperfeiçoar a capacidade de predição e prevenção em saúde pública. Para que esta meta seja alcançada, entretanto, muito trabalho ainda se faz necessário, particularmente a estimação do efeito de cada classe e do próprio processo classificatório na incidência da doença e sua distribuição espaço-temporal.

Os resultados obtidos aqui demonstram que mesmo dispondo-se de recursos limitados, imagens de baixo custo, ampla disponibilidade e média resolução, sistemas abertos e livres para processamento e integração das informações, é possível a obtenção de parâmetros para compor estudos prospectivos de fatores de risco ambientais para a ocorrência de endemias urbanas.

\section{Resumo}

Este artigo apresenta um estudo de caso - aspectos ambientais relacionados à ocorrência da leishmaniose visceral em Teresina, Piauí, Brasil, no periodo de 1993 a 1996 - visando a discutir o uso de um conjunto de métodos aplicados a imagens de sensoriamento remoto em média resolução no estudo de endemias transmitidas por vetores em áreas urbanas. Foram apresentadas as técnicas essenciais a esse tipo de procedimentos: registro, correção geométrica, restauração, fusão, segmentação e classificação. Quatro classificadores, Maxver, Bhattacharya, K-médias e Isoseg, foram comparados utilizando o índice de correlação intraclasse aplicado à proporção de área segundo cada classe nos setores censitários. Os métodos de avaliação utilizados não tiveram o propósito de definir o melhor classificador, mas desenhar cenários de classificação, de forma a reconhecer as classes melhor identificáveis em área urbana. Mesmo dispondo-se de recursos limitados, imagens de baixo custo, ampla disponibilidade e média resolução, sistemas livres para processamento e integração das informações, é possível identificar as características de ocupação do solo de interesse potencial na análise de riscos ambientais para a ocorrência de endemias urbanas transmitidas por vetores.

Sensores Remotos; Leishmaniose Visceral; Doenças Endêmicas 


\section{Colaboradores}

Todos os autores participaram da elaboração do texto e discussão dos resultados. V. R. M. Correia participou da construção do banco de dados e processamentos.

\section{Referências}

1. Correia VRM, Carvalho MS, Sabroza PC, Vasconcelos $\mathrm{CH}$. Remote sensing as a tool to survey endemic diseases in Brazil. Cad Saúde Pública 2004; 20:891-904.

2. Hay SI, Tucker CJ, Rogers DJ, Packer MJ. Remotely sensed surrogates of meteorological data for the study of the distribution and abundance of arthropod vectors of disease. Ann Trop Med Parasitol 1996; 90:1-19.

3. Werneck GL. Spatial approaches to study the epidemiology of visceral leishmaniasis in Teresina, Brazil [PhD Thesis]. Cambridge: Harvard University; 2000.

4. Werneck GL, Rodrigues LJ, Araújo LB, Santos MV, Moura LS, Lima SS, et al. The burden of Leishmania chagasi infection during an urban outbreak of visceral leishmaniasis in Brazil. Acta Trop 2002; 83:13-8.

5. Phinn S, Stanford M, Scarth P, Murray AT, Shyy PT. Monitoring the composition of urban environments based on the vegetation-impervious surface-soil (VIS) model by subpixel analysis techniques. Int J Remote Sens 2002; 23:4131-53.

6. Mather PM. Computer processing of remotely sensed images; an introduction. 2nd Ed. New York: John Wiley \& Sons; 1999.

7. Haralick RM, Shanmuga K, Dinstein I. Textural features for image classification. IEEE Trans Syst Man Cybern 1973; 6:610-21.

8. Tso B, Mather PM. Classification methods for remotely sensed data. London: Taylor \& Francis; 2001.

9. Rede Integrada de Informações para a Saúde. Comitê temático interdisciplinar: dados espaciais em saúde - relatório. Brasília: Organização Pan-Americana da Saúde/Ministério da Saúde; 1999.

10. Instituto Brasileiro de Geografia e Estatística. Censo demográfico 2000: características da população e dos domicílios (resultado do universo). Rio de Janeiro: Instituto Brasileiro de Geografia e Estatística; 2001.

11. Carvalho M, Pina M, Santos S. Conceitos básicos de sistemas de informações geográficas e cartografia aplicados à saúde. Brasília: Organização PanAmericana da Saúde/Ministério da Saúde; 2000.

12. Federov D, Fonseca LMG, Kenney C, Manjunath BS. Automatic registration and mosaicking system for remotely sensed imagery. In: Anais do XI Simpósio Brasileiro de Sensoriamento Remoto. Belo Horizonte: Instituto Nacional de Pesquisas Espaciais; 2003. p. 317-24.
13. Boggione GA, Fonseca LMG. Restoration of Landsat-7 images. http://www.dpi.inpe.br/ leila/ publications/Restoration_of_Landsat-7_Images. pdf (acessado em 02/Ago/2006).

14. Carper WJ, Lillesand TM, Kiefer RW. The use of intensity-hue-saturation transformation for merging spot phanchromatic and multiespectral image data. Photogrammetric Engineering and Remote Sensing 1990; 56:459-67.

15. Bins L, Erthal G, Fonseca LMG. Um método de classificação não supervisionado por regiões. In: Anais do VI Simpósio Brasileiro de Computação Gráfica e Processamento de Imagens. Comunicações. Recife: Sociedade Brasileira de Computação/Universidade Federal de Pernambuco; 1993. p. 65-8.

16. Bins LS, Fonseca LMG, Erthal GJ, Ii FAM. Satellite imagery segmentation: a region growing approach. In: Anais do VIII Simpósio Brasileiro de Sensoriamento Remoto [CD-ROM]. São José dos Campos: Instituto Nacional de Pesquisas Espaciais; 2003.

17. Congalton RG, Green K. Assessing the accuracy of remotely sensed data: principles and practices. New York: Lewis Publishers; 1999.

18. Cohen J. Coefficient of agreement for nominal scale. Educ Psychol Meas 1960; 20:37-46.

19. Fleiss JL. Statistical methods for rates and proportions. New York: John Wiley \& Sons; 1981.

20. Landis J, Koch GG. The measurements of observer agreement for categorical data. Biometrics 1977; 33:159-79.

21. Szklo M, Nieto EJ. Epidemiology beyond the basics. Gaithersburg: Aspen Publication; 2000.

22. Zha Y, Gao J, Ni S. Use of normalized difference build-up index in automatically mapping urban areas from TM imagery. Int J Remote Sens 2003; 24:583-94.

23. Câmara G, Souza RCM, Freitas UM, Garrido J. SPRING: integrating remote sensing and GIS by object-oriented data modelling. Comput Graph 1996; 20:395-403.

24. Cordeiro JP, Amaral S, Freitas UM, Câmara G. Álgebra de geo-campos e suas aplicações. In: Anais do VIII Simpósio Brasileiro de Sensoriamento Remoto [CD-ROM]. São José dos Campos: Instituto Nacional de Pesquisas Espaciais; 2003.

25. Andrianasolo H, Nakhapakorn K, Fages D, Gonzales J, Barbazan P. A methodology in detailed environment mapping for viral disease survey. http:// www.gisdevelopment.net/aars/acrs/1999/ts13/ ts13037.asp (acessado em 02/Ago/2006). 
26. Guindon B, Zhang Y, Dillabaug C. Landsat urban mapping based on a combined spectral-spatial methodology. Remote Sensing of Environment 2004; 92:218-32.

27. Ministério da Saúde. Manual de vigilância e controle da leishmaniose visceral. http://portal. saude.gov.br/portal/arquivos/pdf/manual_leish_ visceral2006.pdf (acessado em 02/Ago/2006).
28. Costa CHN, Pereira HF, Araújo MV. Epidemia de leishmaniose visceral no Estado do Piauí, Brasil, 1980-1986. Rev Saúde Pública 1990; 24:361-72.

Recebido em 03/Out/2005

Versão final reapresentada em 06/Mar/2006

Aprovado em 06/Fev/2007 